Advancing Aviation Security

H. E. Martz, G. P. Roberson, S. G. Azevedo, J. S. Kallman

December 19, 2011 
This document was prepared as an account of work sponsored by an agency of the United States government. Neither the United States government nor Lawrence Livermore National Security, LLC, nor any of their employees makes any warranty, expressed or implied, or assumes any legal liability or responsibility for the accuracy, completeness, or usefulness of any information, apparatus, product, or process disclosed, or represents that its use would not infringe privately owned rights. Reference herein to any specific commercial product, process, or service by trade name, trademark, manufacturer, or otherwise does not necessarily constitute or imply its endorsement, recommendation, or favoring by the United States government or Lawrence Livermore National Security, LLC. The views and opinions of authors expressed herein do not necessarily state or reflect those of the United States government or Lawrence Livermore National Security, LLC, and shall not be used for advertising or product endorsement purposes.

This work performed under the auspices of the U.S. Department of Energy by Lawrence Livermore National Laboratory under Contract DE-AC52-07NA27344. 


\title{
Advancing Aviation Security
}

\author{
Harry E. Martz, Jr., Pat Roberson, Steve Azevedo, and Jeff Kallman
}

IM 550234

LLNL-TR-520912

This document was prepared as an account of work sponsored by an agency of the United States government. Neither the United States government nor Lawrence Livermore National Security, LLC, nor any of their employees makes any warranty, expressed or implied, or assumes any legal liability or responsibility for the accuracy, completeness, or usefulness of any information, apparatus, product, or process disclosed, or represents that its use would not infringe privately owned rights. Reference herein to any specific commercial product, process, or service by trade name, trademark, manufacturer, or otherwise does not necessarily constitute or imply its endorsement, recommendation, or favoring by the United States government or Lawrence Livermore National Security, LLC. The views and opinions of authors expressed herein do not necessarily state or reflect those of the United States government or Lawrence Livermore National Security, LLC, and shall not be used for advertising or product endorsement purposes.

This work performed under the auspices of the U.S. Department of Energy by Lawrence Livermore National Laboratory under Contract DE-AC52-07NA27344. 


\title{
Advancing Aviation Security For FY11 Innovation, Research, and Technology Report
}

\author{
Harry Martz, Pat Roberson, Steve Azevedo, Jeff Kallman
}

\section{$\underline{\text { Introduction }}$}

Air travel is a popular mode of transportation. Each year, more than 600 million people board U.S. flights for business or pleasure. As demonstrated on 9/11, a coordinated terrorist strike on commercial airlines has devastating consequences where many lives could be lost and the nation's economy could be crippled. The Transportation Security Administration (TSA) has worked diligently to enhance security at airports and on flights so that people and cargo can continue to move freely. Today, all passengers and every piece of checked or carry-on luggage are screened before they are permitted on an aircraft. X-ray systems and walk-through metal detectors allow security personnel to identify dangerous metal objects, such as guns and knives. A more complex problem is the detection of explosives, where detection technologies such as multiple-energy X-ray radiography, computed tomography (CT), full body scanners using millimeter wave and X-ray backscatter and other techniques are used to screen for explosives hidden in luggage, in cargo and on the people boarding the aircraft.

Scientists and engineers at the Lawrence Livermore National Laboratory (LLNL) are working with the Department of Homeland Security (DHS), Science and Technology Directorate (S\&T), Explosives Division (EXD) to help improve aviation security by evaluating and enhancing the performance of nextgeneration explosives detection technologies. LLNL is providing subject matter expertise to TSA for their multi-billion dollar procurements of explosive detection system (EDS) for the nation's airports and provides recommendations for process and system improvements. To further improve aviation security, EXD is funding research at LLNL to develop advanced explosives detection technologies that can more accurately discriminate between a wide range of explosives and non-threat materials. One challenge with scanning for explosives is that some nonthreatening materials share similar characteristics with actual threats, leading to false positives or false alarms. When an alarm is generated, security personnel must review the scan images to clear the alarm or manually open and verify the bag's contents, which can increase labor costs and lead to passenger delays.

The Livermore efforts currently focus on CT-based applications. First developed for the medical field as a method for diagnosing disease, X-ray CT has become instrumental in various industrial Non-Destructive Evaluation (NDE) applications. Explosives detection equipment that incorporates CT uses a broadspectrum X-ray beam to capture projections of an object, in particular, the objects inside a piece of luggage. The CT system then applies complex reconstruction algorithms to the projections to produce a threedimensional representation of the luggage and its contents. Automated threat-detection algorithms (also referred to as threat-recognition algorithms) further process the images to separate each item and flag potential threats for further review (see Figure 1). Transportation security officers can then examine these images and the relevant data to determine whether further interrogation is needed. LLNL has also performed research on advanced X-ray carry-on bag inspection systems and is helping to better understand the performance of X-ray backscatter and millimeter wave passenger screening systems. 


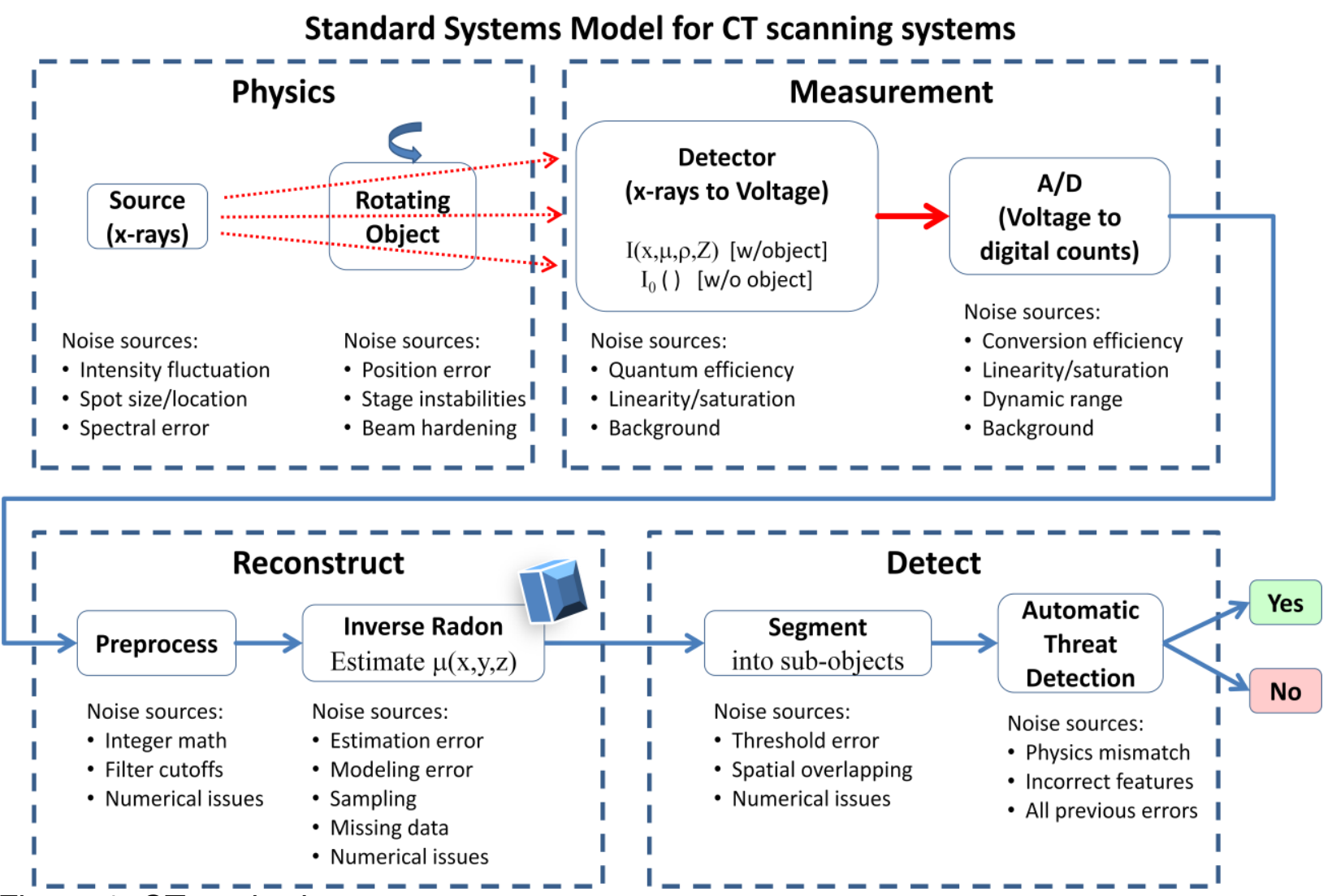

Figure 1: $\overline{\mathrm{C}} \overline{\mathrm{T}}$ method Images obtained from current $\mathrm{C}$ T-based explosives detection systems provide estimates of the linear attenuation coefficients and other features of the items inside luggage. These attenuation coefficients depend strongly on a material's density and elemental composition and on the X-ray source and detector used to measure them.

\section{Engineering Solutions}

The Livermore effort includes the development of a DHS image database that holds a vast catalog of Xray and other properties of explosive threats and non-threat materials. LLNL, in collaboration with the National Explosives Engineering Sciences Security Center (NEXESS), is responsible for defining how explosive test objects are formulated and prepared for use in evaluating the EDS being procured by TSA for U.S. airports. Livermore provides DHS with assistance in establishing quality assurance metrics for collecting data using real explosive specimens designed to evaluate the performance of the vender systems. LLNL is performing research and development on advanced CT algorithms to ensure that they produce precise reconstruction and segmentation results. Livermore is also investigating new source and detector hardware to enhance the performance of existing or next-generation technologies. LLNL is exploring methods to further improve the performance of automated threat-detection algorithms as the quantity of passengers in the U.S. continue to increase and the luggage they pack gets more complex. LLNL is also working with DHS's Center for Excellence (COE) for Explosives Awareness and Localization of Explosives-Related Threats (ALERT) at Northeastern University to help get third parties to look at solutions to explosives detection including image reconstruction, segmentation and automatic threatdetection algorithms.

Livermore is responsible for developing, operating and maintaining the DHS Image Database that provides for storage and the distribution of DHS program data and documents. It is a critical national resource for protecting documents and data so it is available for further research. Data and documents are 
generated at LLNL and other facilities that support the DHS explosive detection efforts. Examples of documents stored or generated in this data base system include program-wide raw data from CT and other explosive detection systems, reconstructed and segmented data generated from the raw data, technical data reports, quality assurance documents, specification documents, and many other documents. Livermore has accumulated approximately 90 terabytes of program data and documents that are stored in this data base.

Livermore is responsible for establishing how explosive formulations are produced and specimens are prepared that are used in evaluating the performance of the various vender explosive detection systems. Formulation preparation data sheets and handling procedures are generated for other labs to use in preparing the specimens. Livermore has two CT systems located in the High Explosive Application Facility (HEAF) that are used to scan real explosives in a safe manner and to validate the formulation process and provide a baseline for quality assurance. Some of the explosives that are being used in this process are sensitive and can only be remotely produced and handled. Livermore scientists and engineers are developing methods for remotely producing, handling and scanning sensitive explosives. One example of a remote handling system is shown in Figure 2. The system is designed so that no pumps or valves are used in the transfer of the explosive for safety reasons. This is accomplished using gravity, low pressure gas and low friction materials for tubing and containers. This system has been successfully used to produce and scan sensitive explosives for DHS. Engineers are currently evaluating other robotic methods for handling additional sensitive materials.

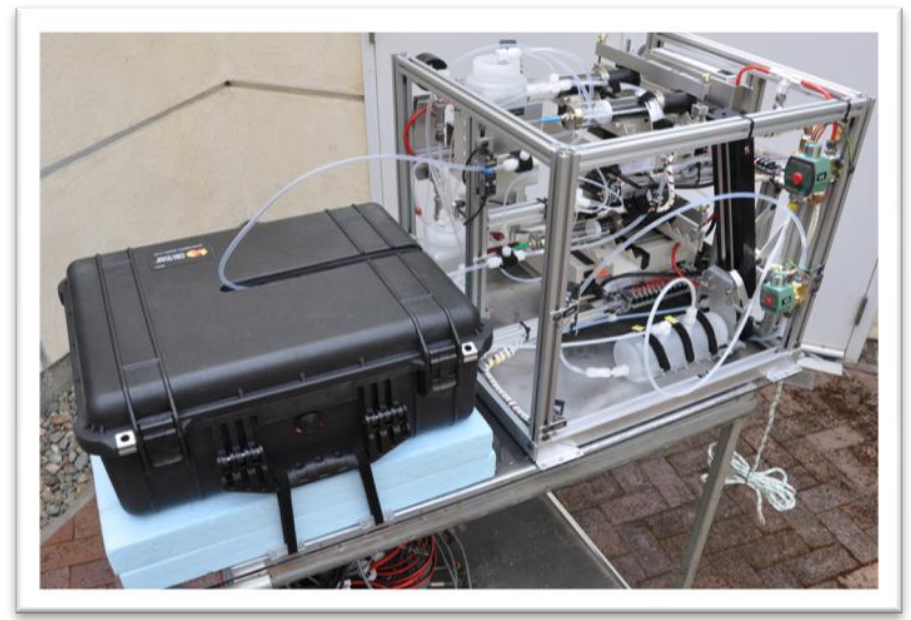

Figure 2: Remote handling system used for the production of sensitive explosives and Pelican case that holds the specimen that is scanned in the CT system.

Livermore engineers are evaluating reconstruction, segmentation and automated threat-detection algorithms to determine how to improve EDS (see Figure 3) and methods for use in advanced X-ray dualenergy techniques. In this detection scheme, the detector measures the linear attenuation coefficients of materials at two X-ray energies, one low in the spectrum and the other high. The two measurements provide a stronger basis for interpreting an object's elemental composition and density, thus improving the system's detection capabilities with fewer false alarms.

At the energies used to scan luggage, $\mathrm{X}$-ray attenuation is determined by three kinds of interaction processes occurring between the X-rays and the object: coherent scattering, photoelectric absorption and Compton scattering. In coherent scattering, incident X-rays are deflected by atoms in an object, whereas in the photoelectric effect, they are completely absorbed. With Compton scattering, the X-ray energy is partially transferred to an electron that is then excited or ejected from an atom. Current detection techniques combine the first two processes into a single parameter. However, it may be possible to obtain a more precise signature of the types of atoms within objects and thus more effectively identify specific explosive materials by measuring all three interactions. Livermore scientists and engineers are using the Laboratory's high-performance computers to model the three interaction processes. These models can artificially adjust the physics parameters, such as X-ray spectra, to improve the understanding of materials and object 
characterization. With that knowledge, the researchers can evaluate the algorithms used in existing explosives detection systems and reconfigure the codes for enhanced performance.

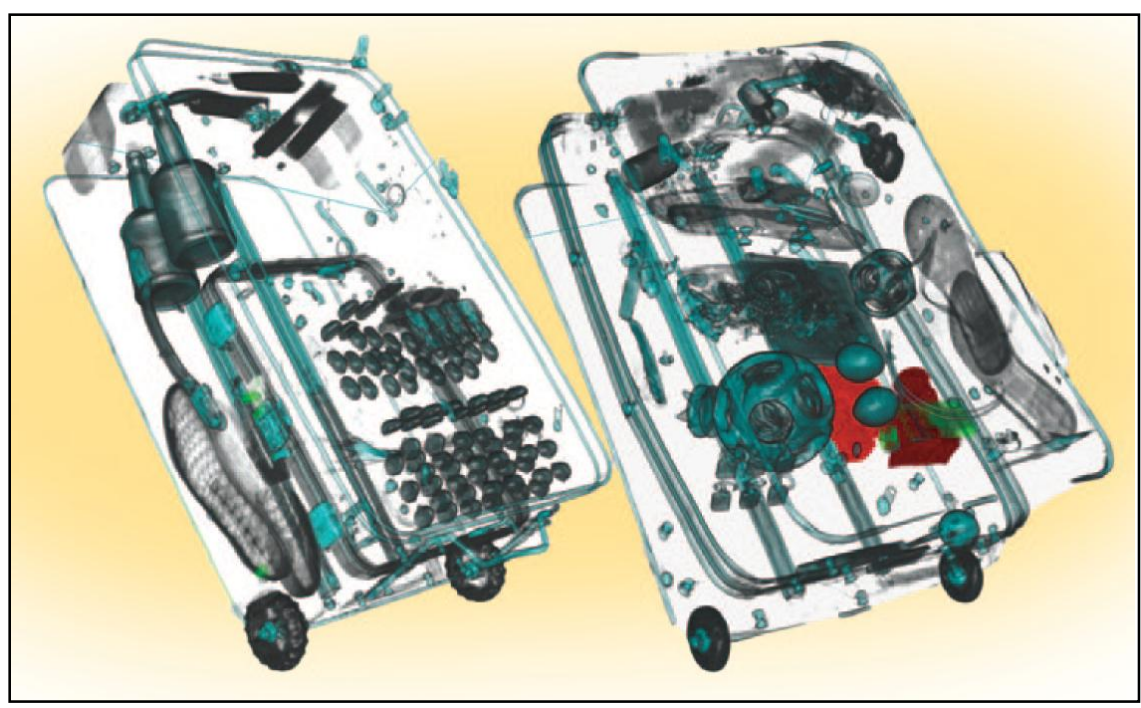

Figure 3: Computed tomography systems produce two- and three-dimensional digital images showing the contents of a bag. Transportation security personnel can review such an image to determine if a threat is present or to clear a false alarm. Areas highlighted in red indicate potential threats. (Courtesy of Safran Morpho Detection.)

Reconstruction algorithms process the projections from CT systems and correct for imperfections in the image quality. In some cases, artifacts, or errors introduced as part of the processing and reconstruction, compromise the image quality. Artifacts such as beam hardening, rings, and streaks can compound the problem. Improved reconstruction algorithms would reduce artifacts in CT images and enhance segmentation to more clearly define objects and their boundaries, increasing threat detection while decreasing the false-alarm rate and thus reducing the intervention required by security personnel.

Segmentation is the process of separating and digitally extracting the objects that make up a bag. Once a bag has been segmented, each object can be examined to determine its features. Examples of features include mass, volume, mean attenuation, and texture metrics. These features are used by automated threatdetection algorithms. A poor segmentation might break an object into pieces or merge multiple objects, thereby making the work of the automated threat-detection more difficult. Livermore scientists and engineers are working on methods of measuring how well an algorithm performs in the segmentation task. These metrics are having a major impact on the development of segmentation algorithms being considered for incorporation into EDSs.

Automated threat-detection algorithms analyze the CT images and extract relevant characteristics such as X-ray attenuation, density, effective atomic number, and mass of different materials in a scanned bag. The system then compares data generated by the algorithms with values of known explosives to classify each material as either a threat or a non-threat. To improve the results produced in such comparisons, LLNL scientists and engineers are redesigning the algorithms to better interpret the complex data, including multiple-energy measurements. The researchers are also developing an expanded database of explosives properties to serve as a reference for the algorithms that process the CT data. The database will also be useful for other researchers working in this field, whether they are at other laboratories, government agencies, or academic institutions or with current or potential industrial partners. These improvements should allow explosives detection technologies to more accurately differentiate threats from non-threats and thus enhance detection capabilities, reduce false-alarm rates, and increase the system's operational efficiency.

Ultimately, the research performed at Livermore and the resulting modifications to existing technologies will allow the DHS Science and Technology's Explosives Division, through the Transportation Security 
Administration, to deploy more efficient detection systems. LLNL scientists and engineers are advancing the technologies used at airports to screen for dangerous materials. Thanks to Livermore's expertise in nondestructive evaluation, high explosives formulation and high-performance computing, airport security personnel are becoming better equipped to stay one step ahead of the nation's adversaries, keeping airline passengers safer in an increasingly hostile world.

Key Words: automated threat-detection algorithm, aviation security, explosives detection, Explosives Division, linear attenuation coefficient, non-destructive evaluation, Transportation Security Administration, X-ray computed tomography (CT).

For further information contact Harry Martz (925) 423-4269 (martz2@IInl.gov). 